\title{
Estilos educativos parentales y resiliencia en adolescentes de la Institución Educativa Pública "Colegio Mayor Secundario Presidente del Perú"
}

Parental educational styles and resilience in adolescents of the Public Educational Institution "Mayor Secundario Presidente del Perú School"

Recibido el 31 de octubre del 2017, aceptado el 30 de noviembre del 2017

Marquina Luján, Román Jesús roman.marquina@usil.pe ORCID: 0000-0002-5542-7234 Universidad San Ignacio de Loyola- Perú
Para referenciar este artículo: Marquina, R. J. (2017). Estilos educativos parentales y resiliencia en adolescentes de la Institución Educativa Pública

"Colegio Mayor Secundario Presidente del Perú". ConCiencia EPG, 2(2), 20-27.

\section{Resumen}

$\mathrm{L}$ a presente investigación de tipo descriptivo-correlacional tuvo como objetivos describir los niveles de resiliencia en los adolescentes del Colegio Mayor Secundario Presidente del Perú y describir los estilos educativos parentales que percibían en sus hogares, así como la relación existente entre dichas variables. La muestra fue de 900 estudiantes, evaluados por las escalas de resiliencia (ER) y de normas y exigencias (ENE-H); estas fueron sometidas al análisis factorial de Kayser-Meyer-Oklin (KMO), dándonos valores de 0.860 para la versión padre y 0.844 para la versión madre; La escala de resiliencia obtuvo un KMO 0.904. Los resultados del test de Bartlett dieron una $\mathrm{p}=0.000$; indicando que existe alta significación. El Alfa de Cronbach dio un valor de 0.722 y 0.706 para la (ENE-H) para padres y madres respectivamente; mientras la fiabilidad de (ER) proporcionó un valor de 0.820; caracterizándolas como altamente confiables. Los resultados indican que con una $\mathrm{p}=0,000$, se demuestra la existencia de relación directa y muy significativa entre la resiliencia y el estilo educativo parental inductivo.

Palabras Clave: estilos educativos parentales, resiliencia, inductiva, rígida, indulgente

\section{Summary}

The present investigation of descriptive-co relational type had as objectives to describe the levels of resilience in the adolescents of the "Mayor Secundario Presidente del Perú School" and the parental educational styles that were perceived in their homes, also the existing relation between these variables.The test was conducted on 900 students, evaluated by the scales of resilience (ER) and standards and requirements (ENE-H); these were subjected to the factorial analysis of Kayser-Meyer-Oklin (KMO), giving us values of 0.860 for the father version and 0.844 for 
the mother versión. The resilience scale obtained a KMO 0.904. The Bartlett test results gave $p=0.000$; indicating that there is high significance. The Alpha of Cronbach gave a value of 0.722 and 0.706 for the (ENE-H) for fathers and mothers respectively; while the reliability of (ER) provided a value of 0.820; characterizing them as highly reliable. The results indicate that with a $\mathrm{p}=0.000$, the existence of direct and very significant relationship between the resilience and the inductive parental educational style is demonstrated.

Key Words: Parental education styles, Resilience, inductive, rigid, indulgent

\section{Introducción}

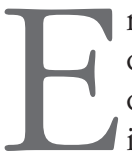
n el Perú y en el mundo entero hablar de cómo la familia favorece al desarrollo de las personas, es un tema de suma importancia, no solo para ellos, sino también para la sociedad en general, debido a que muchos de nuestros comportamientos están cimentados en el hogar; sin embargo, también se debe tener en cuenta que en algunos casos la familia queda muy lejos de ser un ente formador de habilidades, debido a la mala dinámica familiar, producida por la violencia dentro del hogar o la falta de conocimiento para brindar adecuados estilos educativos parentales.

El mundo actual exige estudiantes cada vez más tolerantes a las presiones académicas y sociales, con mayor razón en los países que se encuentran en desarrollo como lo es Perú, se necesitan tener adolescentes con mayor independencia al tomar sus decisiones, jóvenes que puedan arriesgarse a experimentar situaciones nuevas que tengan que ver con la obtención de sus metas u objetivos adecuados, como por ejemplo, concursar para ganar una beca estudiantil, destacar en algún deporte, realizar algún experimento académico, etc. pero muchas veces vemos lo contrario en nuestros jóvenes quienes presentan conductas riesgosas que los llevan al peligro de su integridad física, personal y sexual.
Siempre se considera que la familia es el eje principal del desarrollo humano, en ella se desarrolla la capacidad de afronte que tenemos los seres humanos frente a las distintas circunstancias que nos depara la vida; es decir, en ese ambiente, se desarrollan habilidades para enfrentar problemas y salir airosos a pesar de las adversidades, sino como explicaríamos como mucha gente en situación de pobreza ha podido salir adelante a pesar de las dificultades.

En el año 2015, El Colegio Mayor Secundario Presidente del Perú de Lima, albergaba a un promedio de 900 estudiantes de diferentes zonas del país, siendo en su mayoría adolescentes que viven con bajos recursos económicos y que han estudiado en colegios públicos de su sector, por lo cual podríamos pensar que tampoco han contado con recursos didácticos tecnológicos modernos. Estos jóvenes destacan por su buen desempeño escolar y actitudinal, viven internados dentro del mismo Colegio en Lima, sus familias se encuentran lejos y sin embargo, eso parece no afectarles tanto o al extremo de no caer en episodios depresivos severos por alejamiento que tienen de sus familiares, de allí podríamos preguntarnos qué provoca que estos adolescentes puedan resistir a las presiones de su nuevos contextos y que no solo lo pueden soportar sino que también salir exitosos de ello, por su corta edad y falta de experiencia podríamos inferir como una primera idea que la respuesta estaría en la educación familiar que recibieron en sus hogares.

Lo mencionado anteriormente nos lleva a tratar de llegar a la respuesta del problema planteado y poder establecer si existe alguna relación entre los estilos predominantes de formación familiar y resiliencia.

\section{Método}

Participantes: La población del estudio estuvo conformada por 900 estudiantes varones y mujeres de la Institución Educativa Pública "Colegio Mayor 
Secundario Presidente del Perú", ubicada en Huampaní, perteneciente al Distrito de LuriganchoChosica-Lima, quienes están en edades de entre 13 y 17 años, además, se encuentra cursando tercero, cuarto y quinto grado de educación secundaria.

Instrumentos: Para el presente estudio se utilizó la Escala de Normas y Exigencias para Hijos ENE-H, elaborado por Bersabé, Fuentes, Motrico (2001), que consta de tres dimensiones y 25 ítems. Antes de la aplicación final, los autores hicieron la validez de los ítems (correlación ítem-puntuación total corregida) con valores por encima de 0,40 ; lo que indica un buen comportamiento psicométrico. Para la fiabilidad como consistencia interna resultó bastante elevada encontrando que para el Padre en sus factores I, II y III existía un valor de alfa de Cronbach de 0,85, 0,73 y 0,60 respectivamente; para la escala Madre se halló en los factores I, II y III las puntuaciones de 0,80, 0,72 y 0,64, siguiendo el mismo orden de la dimensión. Estos valores nos demuestran que estas escalas presentan una adecuada consistencia interna. Asimismo, comprobaron que esos coeficientes (Alpha de Cronbach) disminuían si se eliminaba cualquiera de los ítems, significando la importante contribución de cada uno de ellos. Para propósitos de este estudio, creímos conveniente realizar la validez de correlación de ítems test, por tal motivo se aplicó el formato de validación de correlación ítem test del instrumento por cada escala independientemente (padres y madres). Los resultados, indican que el coeficiente de correlación Ítem-test, presenta una correlación positiva en la mayoría de los ítems, obteniéndose correlaciones por encima de ,25; sin embargo, se observa que el ítem 10 en ambas escalas (padre y madres) presentan una correlación ítem-test menor a ,25; criterio mínimo para establecer la homogeneidad de los ítems de un test y su confiabilidad se obtuvo a través del método de consistencia interna, con el coeficiente Alfa de Cronbach, los valores de la escala de estilos educativos parentales de clima familiar aplicada a adolescentes obtuvo un índice Alfa de Cronbach de ,722 para padres y ,706 para madres, lo que indica que el instrumento presenta una adecuada consistencia interna.

El instrumento que se utilizó para evaluar la resiliencia fue la Escala de Resiliencia (ER) de Wagnild y Young (1993) adaptado a nuestro medio por Del Águila (2003), que consta de 25 ítems, esta autora determinó la confiablidad de la escala a través de la consistencia interna, del método Alpha de Cronbach, obteniendo, 7622, y un coeficiente Sperman Brown de 5825; lo cual indica un moderado grado de confiabilidad total. Asimismo, realizó el análisis factorial correspondiente, para explorar el análisis de la validez del instrumento obteniéndose un puntaje Kayser-Meyer-Oklin (KMO) de ,639 y resultados en el Test de Bartlett altamente significativo $(\mathrm{p}<0,001)$.

En la validez de contenido de la escala de resiliencia se efectuó por análisis de correlación e ítem-test, utilizando el coeficiente producto momento de Pearson corregido efectuado en la muestra de estandarización, donde se observa que los ítems evaluados contribuyen al constructo para la medición de la resiliencia. La validación de correlación Ítem-Test nos señalan que el coeficiente de correlación Ítem-test, tiene una correlación positiva en todos los ítems, resultando tener correlaciones por encima de ,25. Y la confiabilidad de la presente escala se obtuvo; a través del método de consistencia interna, coeficiente Alfa de Crombach, el cual nos da el valor de, 820 el cae dentro de los parámetros aceptables para demostrar que la escala es fiable.

Procedimiento: Para la recolección de datos se llevó a cabo con los estudiantes de la Institución Educativa Pública "Colegio Mayor Secundario Presidente del Perú", ubicada en Huampaní, perteneciente al Distrito de Lurigancho- Chosica-Lima; luego de hacer los permisos correspondientes con el director, se pasó a la evaluación respectiva con la administración de los dos instrumentos seleccionados, dicha administración fue realizada de manera presencial y se consideró hacerla en forma grupal, los cuales siendo evaluados casi de manera simultánea en cada aula de clases. A fin 
de brindar las comodidades del caso; la aplicación de las mismas se inició con la escala de resiliencia, seguido de la escala de estilos educativos parentales, con una duración de aproximadamente quince minutos para ambos instrumentos. Posteriormente, luego de la recolección de datos, se procedió con el análisis respectivo para lo cual se utilizaron los estadísticos correspondientes a través del paquete estadístico SPSS. Finalmente se hizo la interpretación de los datos.

\section{Resultados}

Para esta investigación los resultados de los datos obtenidos han sido procesados y analizados utilizando el software SPSS V22, lo que permite generar cuadros estadísticos y gráficos de barras y contraste de las hipótesis mediante niveles de significación $(\bigotimes=0.05)$, utilizando el criterio del valor $\mathrm{p}$.

Tabla 1

Análisis descriptivo de los estilos educativos parentales

\begin{tabular}{lllll}
\hline \multirow{2}{*}{ Estilo } & Padre & & Madre & \\
& $\mathrm{f}$ & $\%$ & $\mathrm{f}$ & $\%$ \\
\hline Inductivo & 748 & 83.1 & 764 & 84.9 \\
Rígido & 99 & 11 & 87 & 9.7 \\
Indulgente & 53 & 5.9 & 49 & 5.4 \\
TOTAL & 900 & 100 & 900 & 100 \\
\hline
\end{tabular}

En la tabla 1, los datos señalan que el estilo educativo que existe un predominio del estilo educativo inductivo en ambos padres (padre 83.1\%; madre 84.9\%), seguido del rígido (padre 11\%; madre 9.7\%) y finalmente, el estilo indulgente (padre 5.9\%; madre 5.4\%).

Tabla 2

Análisis descriptivo de los estilos educativos parentales

\begin{tabular}{lll}
\hline \multirow{2}{*}{ Niveles } & Resiliencia & \\
& $\mathrm{f}$ & $\%$ \\
\hline Bajo & 478 & 53.1 \\
Medio & 240 & 26.7 \\
Alto & 182 & 20.2 \\
Total & 900 & 100 \\
\hline
\end{tabular}

Las puntuaciones obtenidas señalan que la resiliencia expresadas en niveles (bajo, medio, alto). Observamos que el nivel medio y alto suman un 46.9\% a comparación del 53.1\% que se ubica en el nivel bajo, esto motiva a realizar una exploración más profunda de los resultados, por tales motivos se efectuó el análisis descriptivo de las dimensiones que conforman la resiliencia. 


\section{Artículos Empíricos}

Tabla 3

Análisis descriptivo de la resiliencia en los adolescentes, según sus dimensiones

\begin{tabular}{|c|c|c|c|c|c|c|c|c|c|c|}
\hline \multirow[t]{2}{*}{ Nivel } & \multicolumn{2}{|c|}{ Ecuanimidad } & \multicolumn{2}{|c|}{ Perseverancia } & \multicolumn{2}{|c|}{$\begin{array}{l}\text { Confianza en sí } \\
\text { mismo }\end{array}$} & \multicolumn{2}{|c|}{$\begin{array}{l}\text { Satisfacción } \\
\text { personal }\end{array}$} & \multirow{2}{*}{$\begin{array}{l}\text { Sentirse } \\
\text { solo } \\
\mathrm{f}\end{array}$} & \multirow[t]{2}{*}{ bien } \\
\hline & $\mathrm{f}$ & $\%$ & $\mathrm{f}$ & $\%$ & $\mathrm{f}$ & $\%$ & $\mathrm{f}$ & $\%$ & & \\
\hline Bajo & 281 & 31.2 & 421 & 46.8 & 637 & 70.8 & 628 & 69.8 & 796 & 88.4 \\
\hline Medio & 581 & 64.6 & 435 & 48.3 & 225 & 25 & 233 & 25.9 & 67 & 7.4 \\
\hline Alto & 38 & 4.2 & 44 & 4.6 & 38 & 4.2 & 39 & 4.3 & 37 & 4.1 \\
\hline TOTAL & 900 & 100 & 900 & 100 & 900 & 100 & 900 & 100 & 900 & 100 \\
\hline
\end{tabular}

En la tabla 3, se presenta los resultados del análisis de frecuencias de las dimensiones de la resiliencia. Respecto a ecuanimidad y perseverancia, predomina el nivel medio con el $64.6 \%$ y $48.3 \%$ respectivamente, seguido por el nivel bajo con el $31.2 \%$ y $46.8 \%$; por otro lado, vemos que prevalece el nivel bajo en las

dimensiones confianza en sí mismo (70.8\%), satisfacción personal (69.8\%) y sentirse bien solo (88.4\%), todas estas se encuentran nivel medio. Esto nos permite ver que en ellos existe un predominio de la razón y la constancia en la búsqueda de sus objetivos, los cuales lo llevan a su éxito académico.

Tabla 4

Analisis de correlación de Resiliencia y estilos educativos parentales

\begin{tabular}{|c|c|c|c|c|c|c|}
\hline \multicolumn{7}{|c|}{$\begin{array}{l}\text { Madre } \\
\text { Resiliencia }\end{array}$} \\
\hline \multirow{4}{*}{\multicolumn{2}{|c|}{ Inductivo }} & & Inductiva & Rígida & Indulgente & \\
\hline & & $\mathrm{r}$ &, $189^{* * *}$ &,$- 074^{*}$ &,$- 098^{* *}$ &, $0342^{* * *}$ \\
\hline & & $\mathrm{p}$ &, 000 & ,026 & ,003 &, 000 \\
\hline & & $\mathrm{n}$ & 900 & 900 & 900 & 900 \\
\hline \multirow{3}{*}{\multicolumn{2}{|c|}{ Rígido }} & $\mathrm{r}$ & ,002 & $653^{\text {*** }}$ & $123^{\text {** }}$ &,$- 129^{* * *}$ \\
\hline & & $\mathrm{p}$ & ,959 &, 000 &, 000 &, 000 \\
\hline & & $\mathrm{n}$ & 900 & 900 & 900 & 900 \\
\hline \multirow{3}{*}{\multicolumn{2}{|c|}{ Indulgente }} & $\mathrm{r}$ &,$- 102^{* * *}$ &, $124^{* * *}$ &, $718^{* * *}$ &,- 041 \\
\hline & & $\mathrm{p}$ & ,002 &, 000 &, 000 & ,218 \\
\hline & & $\mathrm{n}$ & 900 & 900 & 900 & 900 \\
\hline \multirow{3}{*}{$\frac{0}{\tilde{Z}}$} & & $\mathrm{r}$ & $197^{* * * *}$ &,$- 114^{* * *}$ &,- 057 & 1 \\
\hline & Resiliencia & $\mathrm{p}$ & ,003 & ,001 & ,086 & \\
\hline & & $\mathrm{n}$ & 900 & 900 & 900 & 900 \\
\hline
\end{tabular}


El análisis de correlación que se presenta en la tabla anterior señala que la resiliencia de los adolescentes se correlacionan de manera directa y muy significativa a nivel medio débil con el estilo educativo inductivo que ejerce el padre $(0,342 * *)$; y se correlaciona de manera inversa y significativa a nivel medio débil con el estilo educativo rígido que ejerce del padre $\left(-0,129^{* *}\right)$. Por otro lado, la resiliencia del adolescente se correlaciona de manera directa y significativa a nivel débil con el estilo educativo inductivo que ejerce la madre $\left(0,197^{* *}\right)$; y se correlaciona de manera inversa y muy significativa a nivel débil con el estilo educativo rígido que ejerce la madre. Cabe señalar que la resiliencia de los adolescentes no se ve correlacionada significativamente con el estilo educativo indulgente que ejercen ambos padres.

Estos resultados nos permiten decir que los estilos educativos de los padres y madres inductivos guardan relación directa con la resiliencia de los adolescentes del Colegio Mayor Secundario Presidente de la Republica.

\section{Discusión}

Se pudo encontrar que si existe relación significativa entre ambas variables, predominando la presencia del estilo educativo inductivo con la resiliencia; Iglesias y Romero (2009) realizaron un estudio similar donde pudieron concluir que los estilos educativos negligentes y autoritarios son los que generan en los adolescentes niveles altos de depresión; esto nos permite decir que el estilo educativo inductivo conduce a desarrollar capacidad de afronte a la diversas situaciones que se nos pueda presentar en la vida diaria, siendo mejores en nuestra habilidad para tomar decisiones y a su vez, saber escoger las alternativas correctas que nos permitan tener un adecuado desarrollo personal.

Por otro lado, Domínguez y Carrión (como se citó en Torio, et. al. 2008), a través de su estudio realizado, concluyeron que el estilo inductivo se encuentra muy ligado a las consecuencias de desarrollo favorables y genera mayor capacidad de ajuste a las demandas sociales en los adolescentes. Quispe y Sigüenza (2013), manifiestan que existe relación entre el clima social familiar y la resiliencia en adolescentes; todo esto nos lleva a suponer que mientras los adolescentes tengan padres más inductivos, estos tendrán mayor posibilidad de poder descubrir sus habilidades y ponerlas en práctica en la situación que lo ameriten; también debemos mencionar que pudimos hallar una relación inversa y significativa entre el ser ecuánime y perseverante con el estilo educativo del padre rígido, lo cual se refiere a aquellos padres que conducen hogares en forma autoritaria, sin negociación en establecimiento de normas y castigos sin diálogos previos, provocando que sus hijos adolescentes tengan menos habilidades para afrontar las diversas adversidades de la vida, emitiendo conductas de rebeldía, rompiendo las reglas o de lo contrario asumiendo una postura mucho más pasiva y/o dependiente, prefiriendo aislarse de su plan de vida, por temor al fracaso.

En cuanto al estilo educativo parental de la madre, pudimos hallar que el tipo inductivo se relaciona con la ecuanimidad, perseverancia; lo cual nos permite manifestar que aquellas madres que utilizan el diálogo frecuentemente para orientar, aconsejar y/o castigar y explicar el castigo; desarrollan mejores habilidades en sus hijos para reparar el error en la decisión que puedan haber tomado, para volver actuar sobre la situación siendo razonable y respetuoso del evento o error a reparar.

Podemos ver que en este trabajo existe un predominio del estilo educativo parental inductivo tanto de la madre como del padre, lo cual nos demuestra que estos adolescentes puedan tener mayor predisposición para explorar y desarrollar sus habilidades académicas y a partir de allí trabajar aspectos ligados a la parte intra e interpersonal que no se encuentran muy desarrolladas.

En cuanto a la resiliencia de los adolescentes podemos observar que en promedio existen un 46.9\% de adolescentes con capacidad resiliente entre los niveles medios y altos, lo cual no 
se encuentra como predominio en la muestra estudiada debido a que tiene muchos participantes entre 13 y 14 años y estos adolescentes son aquellos que recién se encuentran en el proceso de adaptación a su nueva experiencia de vida fuera de su hogar procedente; sin embargo, creemos que estos niveles pueden incrementar conforme el tiempo pase, debido a que en la institución educativa donde ellos estudian fortalecen sus habilidades personales a través de cursos y talleres psicoformativos, donde también se involucran a los padres, como ellos lo manifiestan superar los seis primeros meses en el colegio son determinantes para ver si continúan o abandonan el programa.

No obstante, podemos darnos cuenta de que existe un predominio en niveles medios de la habilidad que tienen para actuar de manera responsables, siendo perseverantes en su toma de decisiones, estas características se consideran esenciales en todos los seres humanos para realizar nuestros planes de vida, seguir nuestras metas y cumplir con nuestros objetivos en forma adecuada. Respecto al género de los adolescentes no encontramos relación alguna, tanto en el estilo del padre como de la madre, lo cual nos indica que la mayoría de ellos percibe que las normas

\section{Referencias}

Benito, J., Byrne, Camacho, J., Chávez, S., López, J., Máiquez, L., \& Chávez, S. (2009). Factores que influyen en el pronóstico de recuperación de las familias en riesgo psicosocial: el papel de la resiliencia del menor, donde sus objetivos fueron averiguar si la resiliencia se da cualquiera que sea la gravedad del impacto de la situación sobre el desarrollo del menor. Revista Psicothema, 21(1) 90-96. Recuperado de http://eds.b.ebscohost.com/ehost/pdfviewer/ pdfviewer?vid=7\&sid=f9f9683c-2847-4c67-9c2f7b373f7ca7d4\%40sessionmgr101 se cumplan porque son formas de convivir, sin diferenciar el ser hombre o mujer.

Por otro lado, existe relación en los estilos educativos parentales con que, si el adolescente vive con uno de los padres o con ambos, encontrando que aquellos adolescentes que viven con ambos padres tienden a desarrollar mejores niveles de resiliencia, este resultado se puede comparar con lo hallado por Benito, et al. (2009). Quienes encontraron en su investigación que las familias biparentales presentaron a adolescentes con mayores puntuaciones en la resiliencia.

Por último, no hallamos relación entre el género y la edad con la resiliencia, dicho resultado también fue reportado por Palomino (2011); pero en el caso del género Peña en el 2008 manifestó que las mujeres presentaban mayores fuentes de resiliencia y no encontrando diferencias entre el lugar de procedencia. Además, debemos recalcar que, en aquellos adolescentes que viven con ambos padres tienden a desarrollar mayores niveles de resiliencia, lo cual demuestra lo importante que es el entorno familiar en la formación de todos los seres humanos.

Bersabé, R., Fuentes, M. y Motrico, E. (2001). Análisis psicométrico de dos escalas para evaluar estilos educativos parentales. Psicothema, 13(4), 678-684. Recuperado de http://www.redalyc.org/html/727/72713422/

Del Águila, M. (2003). Niveles de Resiliencia en Adolescentes según género y nivel socioeconómico (Tesis de Maestro en Psicología). Universidad Femenina del sagrado Corazón. Lima, Perú. 


\section{Artículos Empíricos}

Iglesias, B. \& Romero, E. (2009). Estilos parentales percibidos, psicopatología y personalidad en la adolescencia. Revista de psicopatología y psicología clínica, 14(2), 63-77. Recuperado de http:// eds.b.ebscohost.com/ehost/pdfviewer/ pdfviewer?vid=4\&sid=f9f9683c-2847-4c67-9c2f7b373f7ca7d4\%40sessionmgr101

Palomino, A. (2011). Resiliencia en un grupo de estudiantes de primero de secundaria de una institución educativa nacional del departamento de lima y un grupo de estudiantes de primero de secundaria de una institución educativa nacional de Ica (Tesis de Licenciado en psicología). Universidad de San Martin de Porres. Facultad de Psicología. Lima, Perú.
Quispe, C. y Sigüenza, R. (2013). Relación entre clima familiar y resiliencia en adolescentes de un Colegio Nacional del Distrito de San Luis (Tesis de Maestro en Psicología). Universidad Femenina Del Sagrado Corazón. Lima, Perú.

Torio, S., Peña, J. y Rodríguez, M. (2008). Estilos educativos parentales: Revisión bibliográfica y reformulación teórica. Teorías de la educación, (20), 151-178. Recuperado de http://gredos.usal.es/jspui/ bitstream/10366/1805/1/Estilos_educativos_ paren_tales_revisión_b.pdf.

Wagnild, G.M. y Young, H.M. (1993). Development and psychometric evaluation of resilience scale. Journal of Nursing Measurement, 1(2), 165-178. 